\title{
RESPONSE OF NEURONS IN THE CAT'S LATERAL GENICULATE NUCLEUS TO MOVING BARS OF DIFFERENT LENGTH ${ }^{1}$
}

\author{
B. G. CLELAND, B. B. LEE, ${ }^{2}$ and T. R. VIDYASAGAR
}

Max Planck Institute for Biophysical Chemistry, Department of Neurobiology, D.3400 Göttingen-Nikolausberg, Federal Republic of Germany

Received March 1, 1982; Revised June 29, 1982; Accepted July 7, 1982

\begin{abstract}
It is well recognized that in the visual cortex of the cat, some of the cells (hypercomplex) are sharply tuned for the length of a bar moving backwards and forwards across their receptive fields. Other cells (simple) exhibit no such tuning but appear to respond proportionately over a range of bar lengths. The tuning seen in hypercomplex cells is already observable to a lesser degree in retinal ganglion cells. Our experiments were carried out to determine the extent of this tuning in the lateral geniculate nucleus, which relays information from the retina to the cortex. Results show that geniculate cells have tuning properties intermediate between those of ganglion cells and hypercomplex cells. By adding together a linear array of geniculate cells, it is possible to model the characteristics of a simple cell and to demonstrate that while an elongated bar gives a minimal response in hypercomplex cells, it should have little effect on the response of simple cells.
\end{abstract}

Relay cells in the lateral geniculate nucleus of the cat are similar to the majority of ganglion cells in the retina in having antagonistic center-surround properties (Hubel and Wiesel, 1961; Kuffler, 1953; Cleland and Levick, 1974). However, this antagonism seen in the lateral geniculate nucleus is simply a reflection of properties that have already been established in the retina, since the excitatory inputs to geniculate cells all have the same center-surround polarity as that of the geniculate cell (Levick et al., 1972). The main difference lies in the "suppressive field" of geniculate cells (Levick et al., 1972), an inhibitory region, first described by Hubel and Wiesel (1961), that overlaps the ganglion cell surround. Using intracellular recording techniques, McIlwain and Creutzfeldt (1967) and Singer and Creutzfeldt (1970) demonstrated convincingly that the inhibition occurs within the geniculate nucleus, and Dubin and Cleland (1977) have suggested a synaptic circuitry of intrinsic and extrinsic interneurons as the basis of this inhibition. Quantitative experiments have shown that for whole field stimulation, the inhibition can supplement the antagonistic surround of ganglion cells to reduce the center response for an optimal spot by over a log unit (Hubel and Wiesel, 1961; Hammond, 1973).

Whereas the inhibition in the geniculate nucleus tends to be nonspecific, with convergence of both brisk sus-

\footnotetext{
I T. R. V. acknowledges the support of the Alexander von Humboldt Stiftung. B. G. C. was on leave from the Department of Physiology, Australian National University, Canberra, A.C.T. 2600, Australia.

${ }^{2}$ To whom correspondence should be addressed.
}

tained and brisk transient inputs (Hoffmann et al., 1972; Singer and Bedworth, 1973), this does not appear to be the case for excitation where the two classes largely remain separate. It has been shown that relay cells can receive their excitatory input from one to five ganglion cells, with all of the cells being of the same center type, either on center or off center, as the geniculate cell (Cleland et al., 1971a, b). The major portion of the input can readily come from a single ganglion cell or from up to three cells with closely overlapping receptive fields (Levick et al., 1972), so that there is very little loss of spatial information as the signal is relayed through the lateral geniculate nucleus (So and Shapley, 1981).

While geniculate cells respond well to stationary flashing stimuli, such is not the case for cells in the visual cortex, where there is a distinct preference for bars and edges with specific directions of movement (Hubel and Wiesel, 1959, 1962). With stimuli similar to those used to explore cells in the visual cortex, Dreher and Sanderson (1973) were able to demonstrate that many of the excitatory characteristics of cortical cells could readily be discerned in single geniculate cells. These properties have been explored further, along the dimensions of stimulus velocity (Hess and Wolters, 1979), light adaptation (Lee et al., 1977), and orientation (Daniels et al., 1977; Vidyasagar and Urbas, 1982). However, as far as the inhibitory characteristics of cortical cells are concerned, Bishop et al. (1973) have suggested that the field suppression of geniculate cells is relatively ineffective in determining cortical properties.

The aim of our experiments is to determine how the 
responses of cells in the lateral geniculate nucleus change as changes are made to the length of moving bars used to stimulate the cells. From these results, we will show that geniculate inhibition plays a significant role in determining the degree of end zone inhibition seen in members of the simple family of cells (Kato et al., 1978) in the visual cortex.

\section{Materials and Methods}

Adult cats ( 2 to $5 \mathrm{~kg}$ ) were anesthetized with halothane $(4 \%)$ in a gas mixture of nitrous oxide, oxygen, and carbon dioxide (75:22.5:1.5). Following the induction of anesthesia, the halothane concentration was reduced to 1 to $2 \%$ and venous and tracheal cannulae were inserted. The animals were mounted in a stereotaxic frame and a craniotomy was made over the left lateral geniculate nucleus. Following completion of surgery, the halothane administration was discontinued and the animal was immobilized with an injection of gallamine triethiodide. For further anesthesia, the gas mixture was supplemented by 0.5 to $1.0 \mathrm{mg} / \mathrm{kg} / \mathrm{hr}$ of barbiturate, which was added to the infusion of $\sim 5 \mathrm{mg} / \mathrm{kg} / \mathrm{hr}$ of gallamine triethiodide and $0.5 \mathrm{mg} / \mathrm{kg} / \mathrm{hr}$ of $d$-tubocurarine used to maintain paralysis. End tidal $\mathrm{pCO}_{2}$ was kept near $4 \%$ by regulating the depth of ventilation, and the cat's temperature was maintained at $37.5^{\circ} \mathrm{C}$. The electrocardiogram was monitored on an oscilloscope.

The nictitating membrane of each eye was retracted by application of phenylephrine hydrochloride, and the pupils were dilated and accommodation was abolished by instillation of atropine. The eyes were refracted onto a white tangent screen $114 \mathrm{~cm}$ away with contact lenses of appropriate power, and artificial pupils $(4 \mathrm{~mm})$ were mounted immediately in front of the lenses. The positions of the optic nerve heads and areae centrales were backprojected onto the screen.

A tungsten-in-glass microelectrode was inserted into the brain at coordinates A6, L9 and was lowered until the dorsal lateral geniculate nucleus was encountered. The presence of brisk sustained units and the characteristic sequence of eye dominance indicated the electrode location within the geniculate nucleus. After isolation of the activity of a single cell, the receptive field position was plotted with hand-held stimuli and the cell type was identified as sustained or transient according to the criteria of Cleland et al. (1971a). In all cases, the eye that was not providing excitatory input to the cell was covered.

Vertical white bars of $0.1^{\circ}$ width and variable length were formed by a diaphragm in the object plane of a modified slide projector. These were swept horizontally back and forth across a cell's receptive field by deflection of a mirror in the projection beam. A second projector provided a background of $4 \mathrm{~cd} / \mathrm{m}^{2}$ upon which the contrast of the bar could be changed with neutral density filters.

For each bar length, peristimulus histograms were collected for 20 sweeps with the bar centered so that its midpoint passed over the receptive field center. For some cells, different contrasts, velocities, and midpoint offsets were used. Checks of response variability were obtained by repetition of a given stimulus condition from time to time. Histograms consisted of 200 bins for each direction of stimulus movement with a sweep amplitude of $10^{\circ}$. For data analysis, the stored histograms were smoothed with a two-step Gaussian smoothing algorithm and this was used for display purposes and extraction of peak firing rate.

\section{Results}

Single unit recordings were made from the dorsal lateral geniculate nucleus in 10 cats. For each unit, peristimulus histograms were obtained as bright bars, $0.1^{\circ}$ wide and ranging in length from $0.1^{\circ}$ to $15^{\circ}$ were moved backwards and forwards across the cell's receptive field at a velocity of $5^{\circ} / \mathrm{sec}$. The histograms then were used to plot length-response curves. A total of 67 cells were studied, of which 27 had on-center and 22 had off-center brisk sustained properties, while 18 had on-center brisk transient properties. Generally, we found that off-center brisk transient units responded quite poorly to such a stimulus; thus, we did not attempt to study them further. For the majority of cells, a length-response curve was obtained at a contrast of 20 (ratio of bar luminance to background luminance), and for 18 of these cells, a second length-response curve was obtained at a contrast of 3 . Other curves were obtained variously at contrasts of 1,2 , and 10. Qualitatively, the shape of such curves was similar to that of retinal ganglion cells (Cleland et al., 1979): as the length of the bar was increased, there was a steady increase in the response amplitude until a maximum value was reached; further increase in bar length then caused the response to fall to a lower level which was unaffected by additional extension. The initial increase in response was not always obvious at higher contrasts (Fig. $1 B$ ), as even the shortest bars appeared to give a near maximum response. However, the decrease seen at longer bar lengths was affected little by contrast.

The peristimulus histograms from which these curves were derived were also similar in shape to those seen in retinal ganglion cells (Rodieck and Stone, 1965). For oncenter cells, as the bar first moved onto the receptive field surround, there was commonly a slight reduction in firing which gave way to a sharp peak as the bar moved from the surround to the center of the receptive field (Fig. 1A). Then, on leaving the center of the receptive field, the bar commonly caused the cell to stop firing, due to the effect of both removing the stimulus from the center and moving it onto the surround. Both responses are in the same direction and thus supplement one another. Finally, a secondary peak sometimes could be observed as a rebound firing of the cell when the bar left the surround. This secondary peak was most readily observed in brisk sustained units, especially when extended bars were used.

For the off-center brisk sustained cells, the response was largely the complement of the response seen with on-center cells. The first noticeable change in the cell's behavior occurred as the bar moved onto the center of the receptive field and caused a suppression of firing. This suppression gave way to a prominent peak that was a result of the stimulus both leaving the center and moving onto the surround. As with on-center cells, in this situation the two normally antagonistic mechanisms (center and surround) are acting in concert. On leaving the surround, a slight decrease in the response was some- 
A
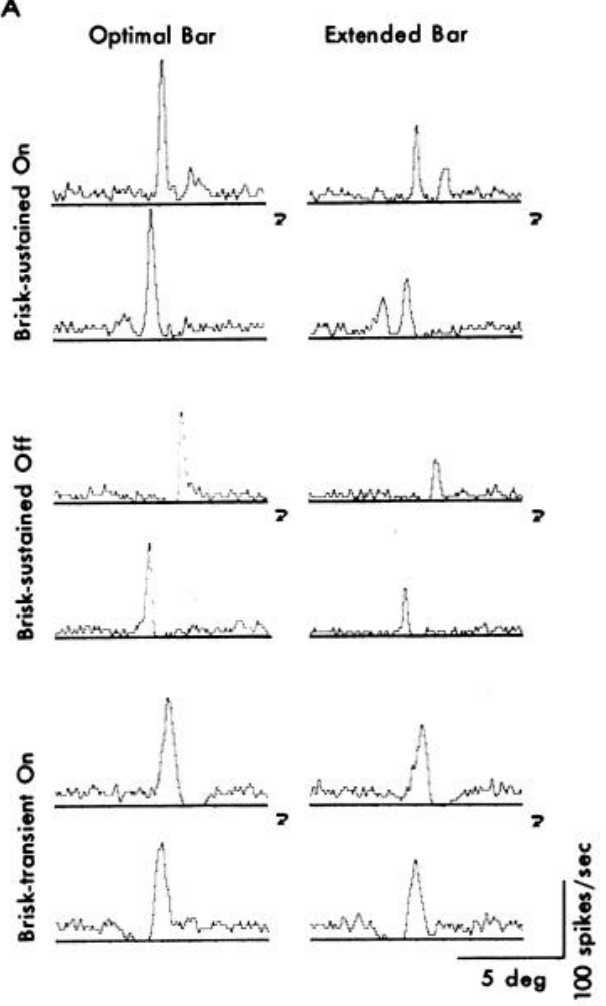

Vel. 5 deg/sec
B
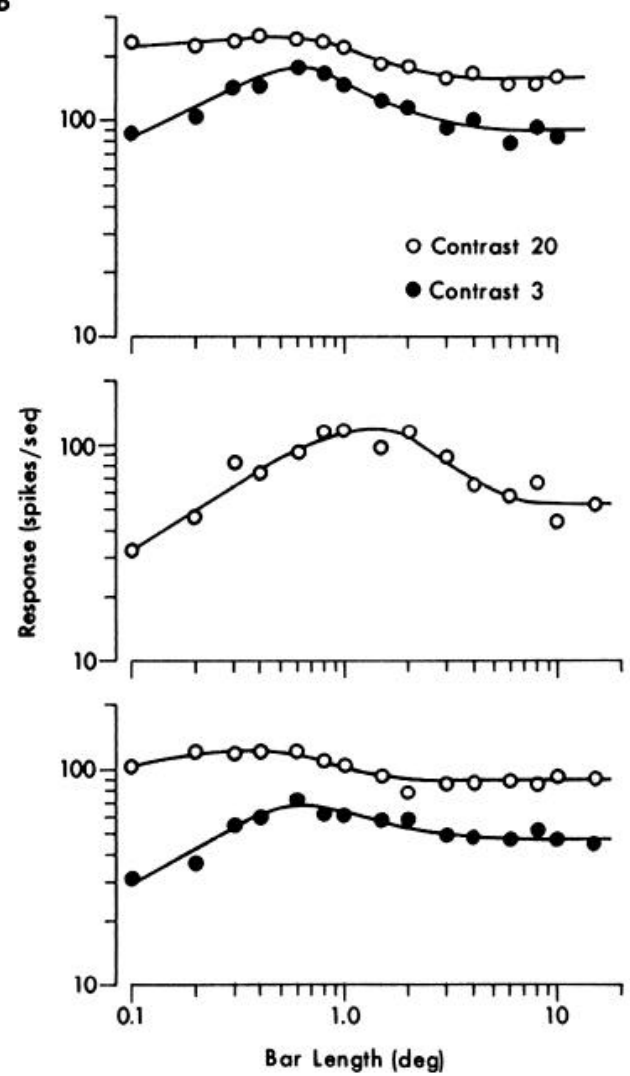

Figure 1. A, Peristimulus histograms for an on-center brisk sustained unit, an off-center brisk sustained unit, and an on-center brisk transient unit. The histograms show both forward motion (left to right on the figure) and return motion (right to left) for a bar producing an optimum response and for an extended $\left(15^{\circ}\right)$ bar. $B$, Length-response curves from which the sample histograms are taken. Background luminance was $4 \mathrm{~cd} / \mathrm{m}^{2}$.

times evident before the cell resumed its normal maintained firing level. The peak observed when the stimulus leaves the center is the one used for plotting lengthresponse curves for off-center cells. Because the center and surround are supplementing one another, no decrease is seen in the response to extended bars when recording from retinal ganglion cells (B. Cleland, unpublished observations). As excitatory effects seen in the geniculate nucleus are a direct reflection of ganglion cell properties, the decrease seen in geniculate cells under these conditions must be due to local inhibitory action within the geniculate nucleus. While the peak used for the measurement of the response of off-center cells represents a characteristic different from that used for the measurement of on-center cells, for cortical cells, bright bars generally have been used (Kato et al., 1978) with little attention being paid to whether they have predominantly on-center or off-center excitation. Thus, for a proper comparison between these two classes of cells (see later), we must ignore this distinction in geniculate cells also.

Separation of the peaks of peristimulus histograms. As the bar moves backwards and forwards, each bin of the peristimulus histogram represents a location on the screen in front of the cat. Thus, the peak of the response occurs each time the bar moves across a specific point on the screen. The separation of the peaks of these responses for each direction of movement is characteristic of the type of receptive field of the unit (Fig. $2 A$ ) and is a result of both spatial factors (receptive field structure) and temporal factors (retinal latency and nerve conduction time). The temporal factors grow in relative importance as the velocity is increased. Among on-center cells, the mean separation was $0.21^{\circ}(\mathrm{SD}=0.39)$ for brisk transient units and $0.53^{\circ}(\mathrm{SD}=0.23)$ for brisk sustained units, while for off-center brisk-sustained cells, the mean separation was $1.54^{\circ}(\mathrm{SD}=0.23)$. This considerably greater separation for off-center cells is to be expected as the peak of the response occurs as the stimulus leaves the center. These kinds of effects may also be seen in simple cells of striate cortex (Lee et al., 1981).

While most cells of a given class fell into a fairly close grouping, there was one on-center brisk sustained cell (Fig. $2 B$ ) that gave a histogram and peak separation typical of an off-center cell. This cell was also unusual in that it had a response reduction with extended bars of $90 \%$, but it showed quite normal characteristics when the velocity was lowered to $2^{\circ} / \mathrm{sec}$.

Response asymmetry and variability. The majority of cells gave a similar response for both directions of movement, but this was not always the case, and two extreme examples of asymmetry are shown in Figure $2 C$, where there is almost a 2 -fold difference in the amplitude of the response for the two directions of movement. For the oncenter cell, the reduction in the primary peak for the return sweep is associated with a considerable enhance- 


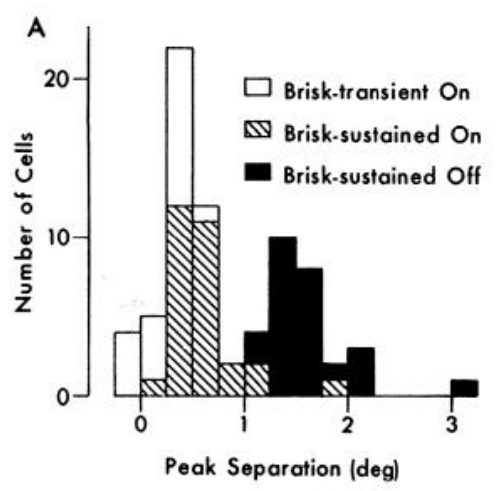

C Brisk-sustained On Brisk-sustained Off

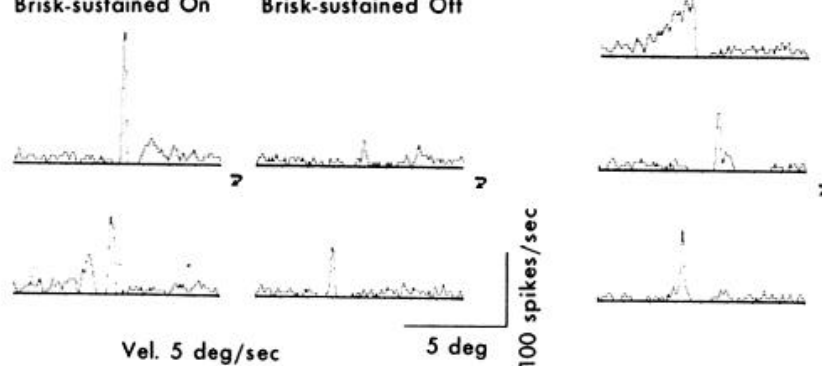

Figure 2. A, Spatial separation of the main peaks of the responses for forward and reverse movement of the bar stimulus at a velocity of $5^{\circ} / \mathrm{sec}$. The smallest separation (mean, $0.21^{\circ}$ ) was seen for on-center brisk transient units, followed by oncenter brisk sustained units (mean, $0.53^{\circ}$ ) and off-center brisk sustained units (mean, $1.54^{\circ}$ ). With one exception, the on- and off-center units show minimal overlap. For this one exception, an on-center brisk sustained unit has a separation typical of offcenter units and the shape of its histogram $(B)$ is also typical of an off-center unit. However, stationary stimuli and responses at lower velocities showed that the unit was clearly in the oncenter class. $C$, Examples of differences seen for the two directions of movement. Although there were a number of clear examples of this, the two responses were, in general, remarkably similar. $D$, Demonstration of one of the difficulties in studying geniculate neurons. There were often apparently spontaneous changes in geniculate inhibition which are seen here for a change in bar length from $0.6^{\circ}$ to $0.8^{\circ}$ but which also could be seen when the histogram was repeated at a given bar length.

ment of the secondary peak, but in neither case is it possible to say whether the asymmetry may not already be occurring within the retina.

Besides this response asymmetry, an additional difficulty that may be encountered in studying cells in the geniculate nucleus is the apparent spontaneous changes in geniculate inhibition. This is shown quite distinctly in Figure $2 D$, where an increase in bar length from $0.6^{\circ}$ to $0.8^{\circ}$ caused little change in the response amplitude but a significant change in the shape of the histogram. Although in this case, the change was associated with an increase in the length of the bar, it is not unusual for such changes to occur when the histograms are repeated at a given bar length.

Comparison of geniculate cell with ganglion cell responses. An obvious difference in geniculate cells compared to retinal ganglion cells is their low maintained firing. This is seen clearly in Figure 3, where results are shown from a geniculate cell which had a strong and easily discriminable prepotential. Such prepotentials are a direct reflection of the afferent spike activity (Bishop et al., 1958; Cleland et al., 1971b) and in this case, the constant amplitude of the prepotential and its maintained activity of 40 spikes/sec indicated that it came from a single brisk sustained ganglion cell. Since all geniculate spikes were preceded by such a prepotential, it can be argued that this ganglion cell was the only significant excitatory input. A similar situation has been analyzed by Hubel and Wiesel (1961) and Singer et al. (1972) who used stationary flashing stimuli.

Length-response curves for both the prepotential and the geniculate spike are shown in Figure $3 B$. The difference between the two can be taken as a measure of the geniculate inhibition and it can be seen that there is a steady increase in inhibition for bar lengths up to approximately $3^{\circ}$. Thus, the inhibitory region would appear to pass through the center of the receptive field and have a diameter of $3^{\circ}$. This would compare with a center size of $0.7^{\circ}$ and a retinal surround size of 1.5 to $2^{\circ}$, based respectively on the bar lengths at which the prepotential length-response curve reaches a peak and first reaches the final plateau level. From Figure $3 C$ it can be seen that, of the spikes resulting from small bars crossing the center of the ganglion cell receptive field, almost $80 \%$ are relayed by the geniculate cell to the visual cortex, whereas for an elongated bar, this is reduced by one-half to $40 \%$. For the unstimulated maintained discharge, only $8 \%$ of the ganglion cell spikes appear at the geniculate output.

From the length-response curves, we have calculated the response reduction with extended bars as the percentage of decrease from the peak of the curve to its final plateau level, and the distribution of these values for all cells is shown in Figure 4. The mean value for on-center brisk sustained units of $53 \%$ ( $\mathrm{SD}=17 \%$ ) is not significantly different $(p \geq 0.05)$ from the value for on-center brisk transient units of $45 \%$ ( $\mathrm{SD}=20 \%$ ), giving an overall mean value of $50 \%$ for on-center cells. For off-center brisk sustained units, the mean response reduction is $41 \%$ (SD $=13 \%)$. From similar experiments, on-center retinal ganglion cells were found to have a response reduction with extended bars of $25 \%$ (Cleland et al., 1979); for off-center cells the response reduction is not significant (see above). Thus, it is possible to calculate the incremental effect of response reduction at the geniculate nucleus as $33 \%$ for on-center units ${ }^{3}$ and $41 \%$ for off-center units; values that are not significantly different.

Relationship to cortical cells. The simple family of cortical cells, consisting of simple and hypercomplex I cells (Kato et al., 1978) or S and $S_{H}$ cells (Henry, 1977), are postulated to represent the earliest stages of processing in the visual cortex (Hubel and Wiesel, 1962; Bullier and Henry, 1979). It is therefore informative to compare

\footnotetext{
${ }^{3}$ If the average response reduction for on-center ganglion cells is $25 \%$, then for an extended bar, $75 \%$ of the response generated by the receptive field center will appear at the ganglion cell output, while only $50 \%$ of the response from the center appears at the geniculate cell output. Thus the geniculate nucleus transmits $67 \%$ of the spikes it receives ( $50 \%$ to $75 \%$ ) and the actual response reduction by the geniculate nucleus will be $33 \%$.
} 
A
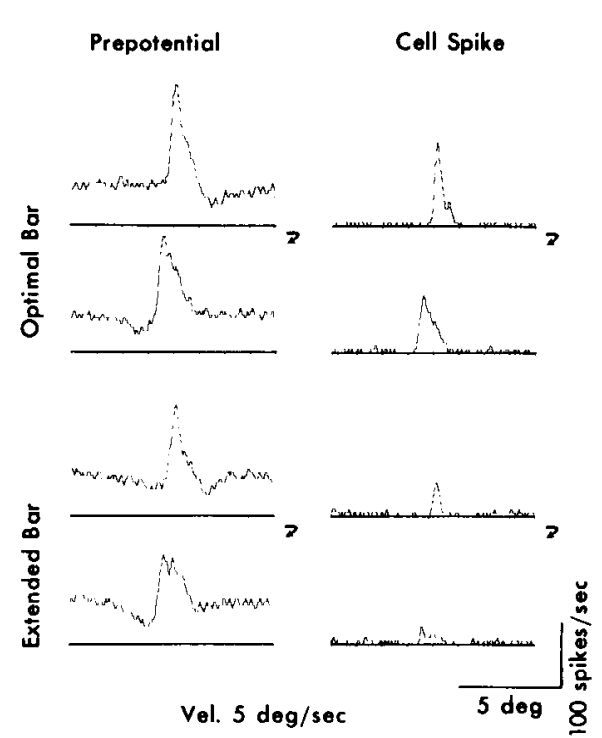

B

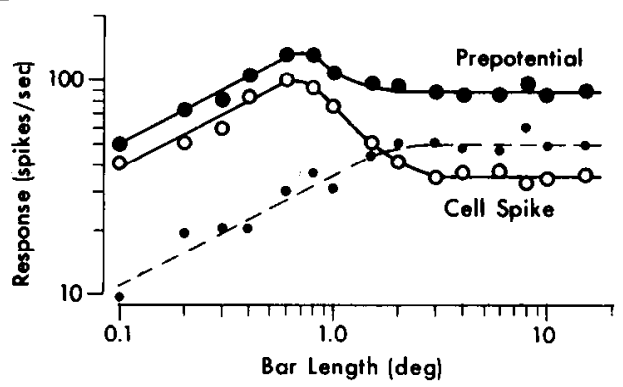

C

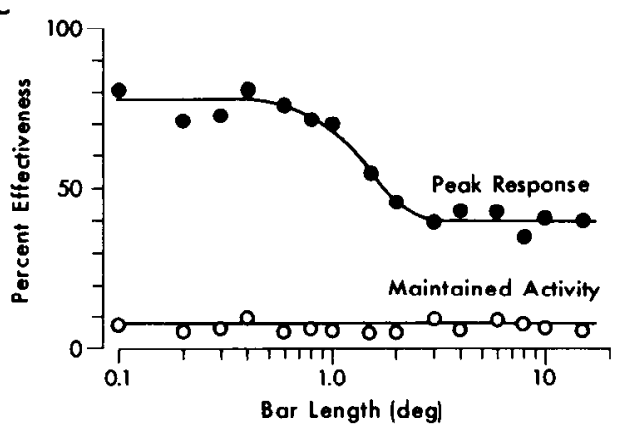

Figure 3. Length-response characteristics of a geniculate cell compared to its retinal input. The geniculate unit had a prominent prepotential which, it is argued, came from a single ganglion cell, and this ganglion cell provided the sole excitatory input to the cell. $A$, Sample histograms for an optimal and an extended $\left(15^{\circ}\right)$ bar. $B$, Length-response curves for the prepotential and cell spike and the arithmetic difference between the two (small solid points and dashed curve). $C$, Percentage of effectiveness of the maintained activity and response peak. The effectiveness is obtained by dividing the geniculate response, or activity, by the prepotential response, or activity.

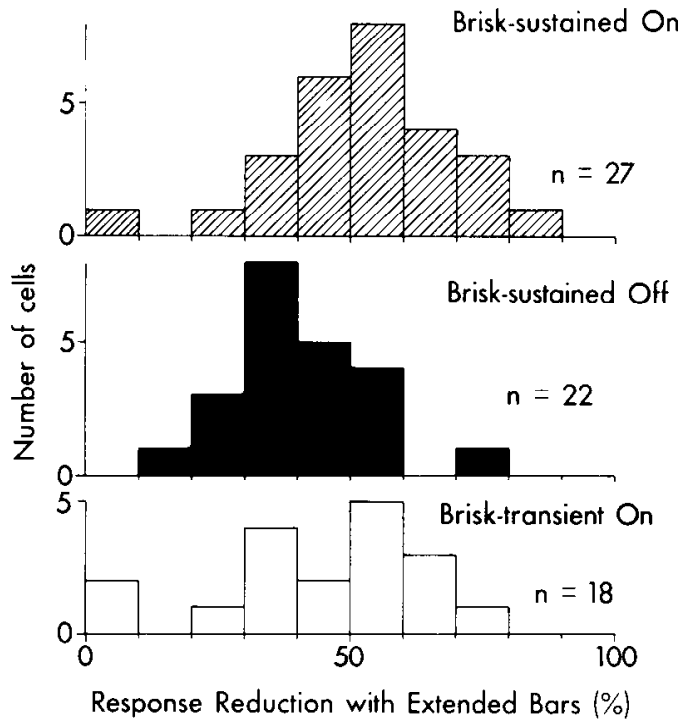

Figure 4. Frequency histograms of the response reduction with extended bars for each of the three cell groups studied. The mean values are $53 \%$ for the on-center brisk sustained unit, $41 \%$ for the off-center brisk sustained unit, and $45 \%$ for the oncenter brisk transient unit.

the properties of these cells with those in the lateral geniculate nucleus. Figure 5 shows a plot of optimal stimulus length as a function of response reduction with extended bars for cortical data replotted from Kato et al. (1978) and compares their data with similar results we have obtained from geniculate cells. For both sets of data, the background was the same and the stimuli used were narrow bars of similar contrast, though no mention is made of the stimulus velocity at which the cortical data were collected. The cortical cells with properties most similar to those of geniculate cells are in the hypercomplex I group, though it can be seen that the geniculate cells tend to have smaller optimal lengths and less response reduction with extended bars.

Comparison with hypercomplex I cells. In comparing our results with those obtained from retinal ganglion cells, we have used the same measure of response; that is, the amplitude of the peak above the maintained firing level. However, in evaluating similar experiments on cells in the visual cortex, Kato et al. (1978) have used the total number of spikes in the response. Such a measure is more readily made in the cortex where there is negligible, or zero, maintained activity. Using the same measure as for cortical cells, we have re-evaluated our data from geniculate cells. For each of the three classes, the two methods of evaluation gave mean values that differed by less than $3 \%$ and hence showed no significant difference. The average response reduction with extended bars for all geniculate cells was $51 \%(\mathrm{SD}=18 \%)$ which compares with a value of $70 \%$ for hypercomplex I cells in the visual cortex (Kato et al., 1978). This implies that the incremental effect in the visual cortex for hypercomplex I cells is $39 \%$.

Comparison with simple cells. Simple cells with a mean optimal stimulus length of $6.4^{\circ}$ and response reduction with extended bars of $7 \%$ (Kato et al., 1978) have properties considerably different from those of geniculate cells, which have a mean optimal stimulus length of $0.9^{\circ}$ and response reduction with extended bars of $51 \%$. As proposed by Rose (1979), such simple cells can be modeled by summing the outputs of several geniculate 


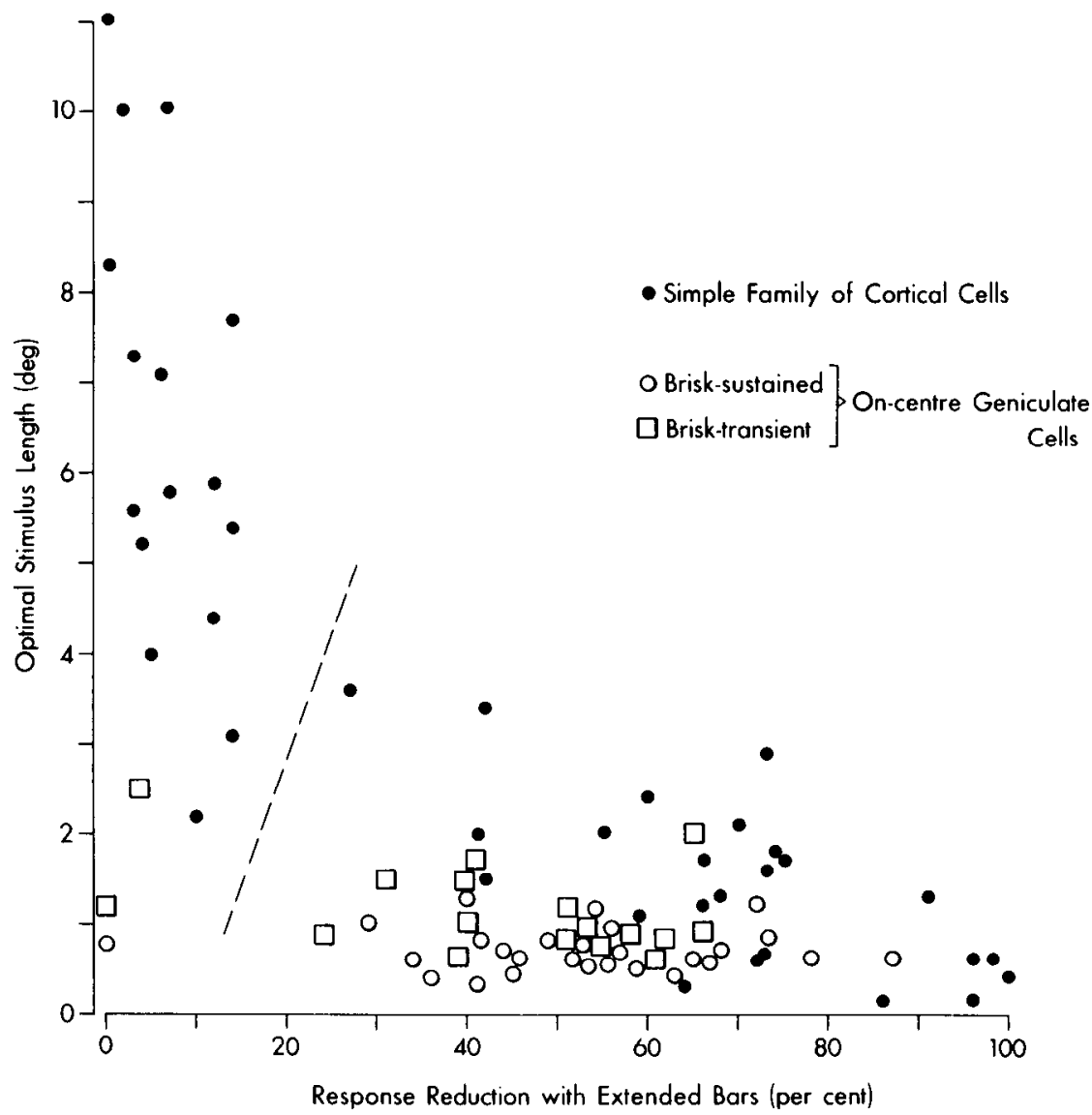

Figure 5. Plot of optimal stimulus length against response reduction with extended bars for on-center geniculate cells. These are compared with similar results for the simple family of cortical cells from Kato et al. (1978). The dashed line divides the cortical cells into simple (to the left) and hypercomplex (to the right) categories. Hypercomplex cells typically have greater response reduction and slightly larger receptive fields than geniculate cells.

cells which are aligned in a row. He considered the situation of the cortical cell having five geniculate inputs as subunits with each subunit having equal strength and with their sensitivity profiles composed of the sum of two Gaussian curves. The five subunits initially were superimposed, and he noted that as they were systematically separated, there was a steady decrease in the degree of response reduction with extended bars coupled with the expected increase in optimal stimulus length, as would be required for an adequate model of simple cells.

Our aim in the following is to look at additional features of such a model using results obtained directly from geniculate cells. In particular, we will consider how the degree of response reduction with extended bars varies as the number of subunits (at fixed spacing) varies and also provide examples of how the shape of the lengthresponse curve is affected by using differing strengths for the subunits.

If we consider a simple cell consisting of a linear array of seven geniculate subunits (Fig. 6), then the relationship of the bar stimulus to each of the subunits will be equivalent to a single subunit with the bar stimulus moving backwards and forwards along each of seven parallel tracks. The spacing between these tracks will be equal to the spacing between the subunits. Symmetry would dictate that only four of the conditions need to be investigated.
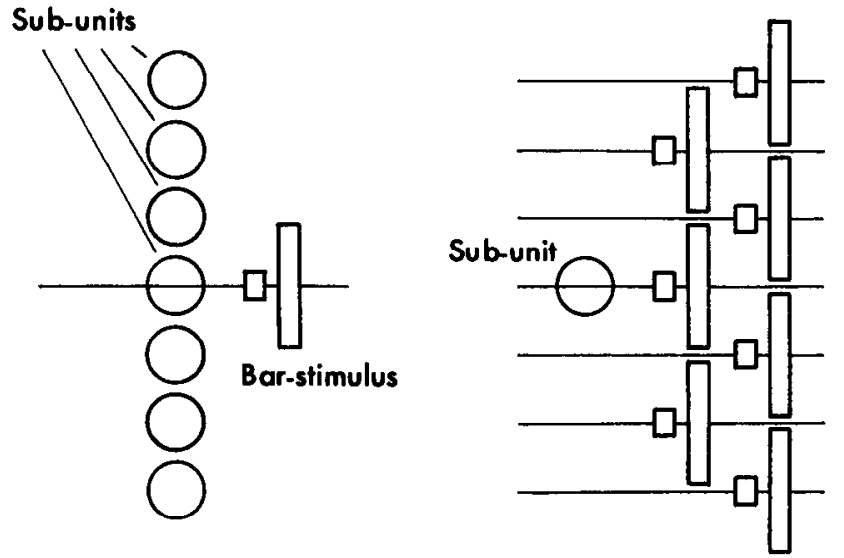

Figure 6. Diagram showing that a bar stimulus moving over a linear array of 7 subunits can be modeled by a single subunit with the bar moving across the center of the subunit and with three distinct (paired) offsets.

Three cells were studied in detail by obtaining lengthresponse curves when the center of the stimulus bar moved through the center of the receptive field and at various offsets. The results of such a series of experiments, with offsets of $0.5^{\circ}$ and $1.0^{\circ}$, are shown for one of these cells in Figure $7 A$ and are consistent with our knowledge of the receptive field structure of cells in the 
A

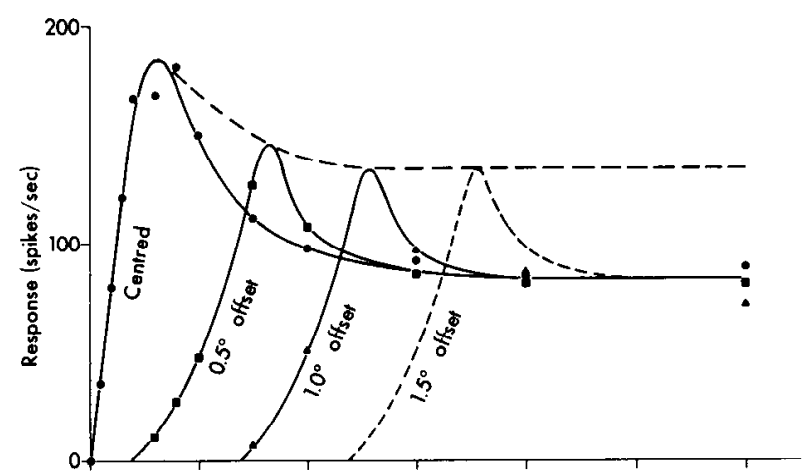

B

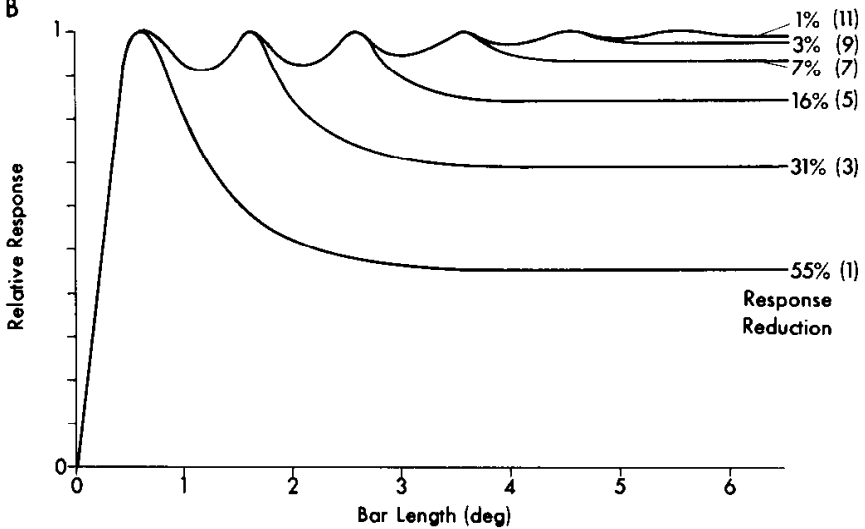

Figure 7. A, Length-response curves for a geniculate cell with the stimulus bar centered on the cell and with offsets of $0.5^{\circ}$ and $1.0^{\circ}$. For offsets of an additional $0.5^{\circ}$, the expectation is for the curve at an offset of $1.0^{\circ}$ to be moved $1.0^{\circ}$ to the right. $B$, Centered length-response curve for the unit in $A$ an expected length-response curves for 3 to 11 subunits separated by $0.5^{\circ}$ with the weighting of the subunits such as to give minimum optimal length and minimum response reduction. The number of subunits is given in parentheses. The curves have all been normalized to give a peak value of 1 . Note that the response reduction decreases with increasing numbers of subunits.

lateral geniculate nucleus. While data points did not always fall near the points of the curves when different offsets were used, the characteristics observed across all three cells allowed us to make a reasonable estimate of these peaks. For a $1.0^{\circ}$ offset, the optimal bar length is approximately $2.5^{\circ}$. At this bar length and offset, the stimulus largely will pass through the whole of the antagonistic surround and the inhibitory region on one side of the receptive field, and hence we would not expect the response to be as strong as for a centered bar of optimal length, nor would we expect further offsets to have additional impact on the optimal response as the end of the stimulus furthest from the receptive field will be having no additional effect. Thus, for an offset of $1^{\circ}$, the response reduction with extended bars is $38 \%$ compared to the response reduction of $55 \%$ for a centered bar. For each $0.5^{\circ}$ increase in offset, the length-response curve obtained at an offset of $1.0^{\circ}$ should be moved along the abscissa by an additional $1.0^{\circ}$.

Figure $7 B$ shows the modeled length-response curves for a simple cell; the curves are representative of $3,5,7$, 9 , and 11 subunits with each of the subunits separated from its neighbor by $0.5^{\circ}$. The weighting of these subunits in each case is such as to give the same optimal stimulus length as for the geniculate cell. It can be seen that once the number of subunits reaches seven, the response reduction with extended bars (7\%) is typical of a simple cell. Closer spacing of subunits would remove the oscillation from the length-response curve but would result in greater response reduction. Alternatively, by increasing the separation to $1^{\circ}$ or greater, the same amount of response reduction could be achieved with fewer subunits but with substantial increase in the oscillation of the curve.

Appropriate weighting of the subunits can provide a range of length-response curves, and three examples are shown in Figure 8. In each case, there are 11 subunits at $0.5^{\circ}$ spacing, and the relative weightings of the subunits are shown as insets for each curve. Figure $8 A$ demonstrates a linear length-response curve with an optimal length of $4.5 \%$. The central subunits have lower weighting than those toward the end. Within limits, this profile could be changed to more uniform weighting by placing greater separation between the central subunits and less separation between the end subunits. Figure $8 B$ demon-
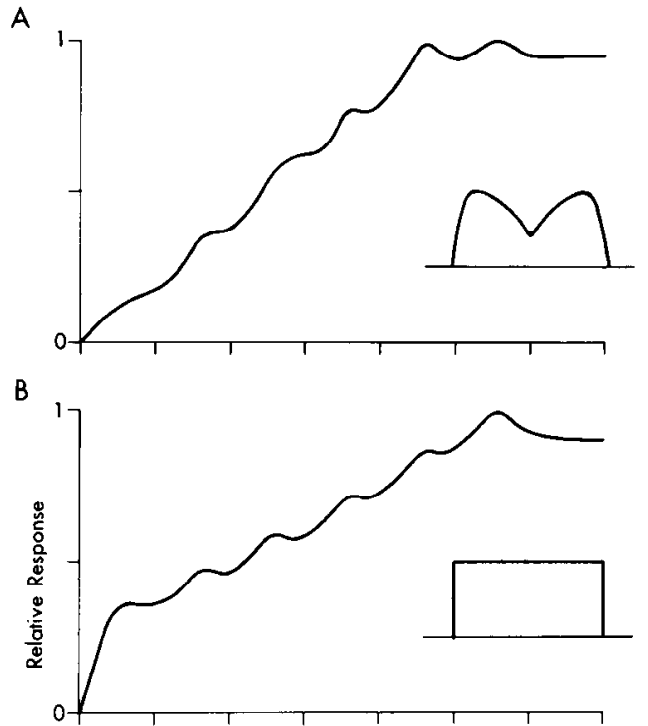

C

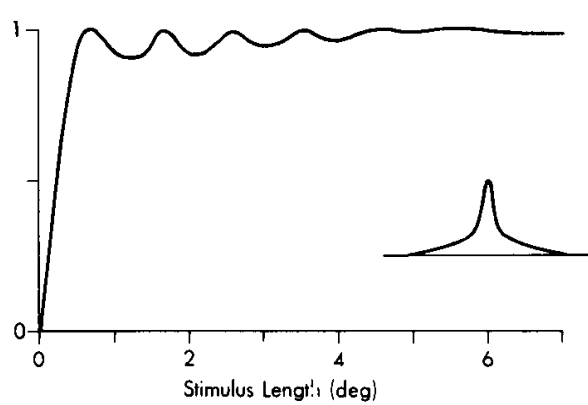

Figure 8. Predicted length-response curves for 11 subunits with spacing of $0.5^{\circ}$. The weighting of each subunit in $A$ to $C$ has been determined to show three distinct conditions. $A$, The weighting has been set to provide a "linear" increase in response with bar length to beyond $4^{\circ} . B$, Equal weighting of all subunits. $C$, The condition in Figure $7 B$ which gives minimum optimal length and minimum response reduction. The envelope of the weighting for the subunits is shown in an inset for each curve. 
strates the effect of giving equal weighting to all subunits and Figure $8 C$ shows the same curve as in Figure $7 B$ for 11 subunits. What is immediately obvious is that lateral extension has produced a simple receptive field, with the greatest amount of response reduction when the end subunits have greatest weight. In all three cases, this lateral extension is approximately $6^{\circ}$, but the optimal length varies from $5.5^{\circ}$ (Fig. $8 B$ ) down to $0.65^{\circ}$ (Fig. $8 C$ ), which is the same as the optimal length of any individual subunit. Thus, linear extension of the receptive field only determines the optimal length through the shape of the receptive field profile.

In many ways the division of the simple family of cells into two distinct groups is unnecessary. Across the population of these cells (Fig. 5) there is an inverse relationship between optimal length and response reduction with extended bars. We would suggest that cortical cells with small receptive fields are excited by a single geniculate cell or a small number of geniculate cells with closely overlapping receptive fields. In either case, the lengthresponse curve will be similar to that for a single geniculate cell. Further, if we assume a constant profile for the receptive field, the linear extension of the field, by adding the excitation from additional geniculate cells, will produce a progressive increase in the optimal length with a concomitant decrease in the response reduction with extended bars.

\section{Discussion}

The goal of our experiments has been to investigate the role of the lateral geniculate nucleus in determining cortical properties. Within the limits of the stimulus parameters so far studied, it is obvious that the inhibition observed in the geniculate nucleus when combined with the surround antagonism of retinal ganglion cells will provide a significant portion of the end zone inhibition (response reduction with extended bars) seen in hypercomplex I cells in the visual cortex. It is difficult to relate this finding to the results of Orban et al. (1979), who concluded that end zone inhibition is stimulus orientation dependent and hence is a purely cortical phenomenon. A satisfactory demonstration of such an effect in the presence of excitation that is, itself, stimulus orientation specific is obviously difficult and may be misleading if there is significant overlap of the two regions. One of the supporting arguments put forward by Orban et al. (1979) against the geniculate nucleus having a significant effect was the presence of "end-free" cells in the cortex, but as we have demonstrated, in agreement with Rose (1979), the lack of end zone inhibition in elongated receptive fields is a natural consequence of such elongation.

We have concluded that of the end zone inhibition seen in hypercomplex cells, $25 \%$ represents a retinal phenomenon (if the stimulus contrast is appropriate for center excitation), $37 \%$ represents a geniculate phenomenon, and $39 \%$ represents a cortical phenomenon. We have expressed these effects in percentage terms, and while this is convenient and allows comparison with other results in the literature, such a measure implicitly considers each of the phenomena as a divisive mechanism. It is far more likely that the surround of a ganglion cell subtracts from the center and that geniculate and cortical inhibition subtract from the excitatory input. Thus, we can also look at these effects in terms of subtractive measures. If the center mechanism of a ganglion cell were to produce a peak firing of 100 spikes/sec, then the surround antagonism should reduce it by 25 spikes/sec, the geniculate inhibition should reduce it by 28 spikes/sec, and the cortical inhibition should reduce it by 18 spikes/sec. The cortical effect viewed in this way would appear to be the weakest of the three.

There are two quite distinct mechanisms that could provide this cortical contribution to the end zone inhibition. One would be a specific visual inhibition localized to the end zones of the receptive field, while the other would be a threshold phenomenon resulting from a generalized inhibition of visual or nonvisual origin. Discrimination between the two possibilities would require a quantitative analysis of cortical properties. The case for a threshold effect has been argued by Henry et al. (1978), who demonstrated for a simple cell that the activity profile of the cell could only be related to a series of length-response curves by the assumption of such a phenomenon. The threshold level required was 5.3 spikes/ sweep compared to a maximum response of 28 spikes/ sweep. It is not possible to compare their results directly in terms of firing rate, nor is it possible to generalize with such a small sample size; nevertheless, it is obvious that additional work must be carried out to demonstrate a direct cortical contribution to end zone inhibition. One approach to this question is that used by Sillito and Versiani (1977), who applied the GABA antagonist bicuculline directly to the cortical cell being studied. They found that there was some reduction in the length preference of the cells, but again the results are qualitative, making it difficult to separate between the two mechanisms. However, for one of the two cells described, it was obvious that for even an optimal bar there was a substantial increase in response when the bicuculline was applied, suggesting that the inhibition had not been limited to the end zones.

We have shown that the length-response characteristics of simple cells can be modeled adequately by a linear array of geniculate cell inputs. However, we equally could have used hypercomplex I cells as the input subunits or a mixture of both. The main effect of using hypercomplex I cells rather than geniculate cells would be to require a greater number of subunits to provide the same degree of end zone inhibition, assuming, of course, an average end zone inhibition of $70 \%$. If all the hypercomplex I cells acting as subunits were to have $100 \%$ end zone inhibition, the simple cell must have $100 \%$ end zone inhibition no matter how many subunits are postulated.

\section{References}

Bishop, P. O., W. Burke, and R. Davis (1958) Synapse discharge by single fibre in mammalian visual system. Nature 182: 728-730.

Bishop, P. O., J. S. Coombs, and G. H. Henry (1973) Receptive fields of simple cells in the cat striate cortex. J. Physiol. (Lond.) 231: 31-60.

Bullier, J., and G. H. Henry (1979) Ordinal position of neurons in cat striate cortex. J. Neurophysiol. 42: 1251-1263.

Cleland, B. G., and W. R. Levick (1974) Brisk and sluggish concentrically organized ganglion cells in the cat's retina. J. 
Physiol. (Lond.) 240: 421-456.

Cleland, B. G., M. W. Dubin, and W. R. Levick (1971a) Sustained and transient neurones in the cat's retina and lateral geniculate nucleus. J. Physiol. (Lond.) 217: 473-496.

Cleland, B. G., M. W. Dubin, and W. R. Levick (1971b) Simultaneous recording of input and output of lateral geniculate neurones. Nature New Biol. 231: 191-192.

Cleland, B. G., T. H. Harding, and U. Tulunay-Keesey (1979) The response of ganglion cells to moving bars of different length. Proc. Aust. Physiol. Pharmacol. Soc. 10: 117P.

Daniels, J. D., J. L. Norman, and J. D. Pettigrew (1977) Biases for oriented moving bars in lateral geniculate nucleus neurons of normal and stripe-reared cats. Exp. Brain Res. 29: 155-172.

Dreher, B., and K. J. Sanderson (1973) Receptive field analysis: Response to moving contours by single lateral geniculate neurones in the cat. J. Physiol. (Lond.) 234: 95-118.

Dubin, M. W., and B. G. Cleland (1977) Organization of visual inputs to interneurons of lateral geniculate nucleus of the cat. J. Neurophysiol. 40: 410-427.

Hammond, P. (1973) Contrasts in spatial organization of receptive fields at geniculate and retinal levels: Centre, surround and outer surround. J. Physiol. (Lond.) 228: 115-137.

Henry, G. H. (1977) Receptive field classes of cells in the striate cortex of the cat. Brain Res. 133: 1-28.

Henry, G. H., A. W. Goodwin, and P. O. Bishop (1978) Spatial summation of responses in receptive fields of single cells in cat striate cortex. Exp. Brain Res. 32: 245-266.

Hess, R., and W. Wolters, (1979) Responses of single cells in cat's lateral geniculate nucleus and area 17 to the velocity of moving visual stimuli. Exp. Brain Res. 34: 273-286.

Hoffmann, K. -P., J. Stone, and S. M. Sherman (1972) Relay of receptive field properties in dorsal lateral geniculate nucleus of the cat. J. Neurophysiol. 35: 518-531.

Hubel, D. H., and T. N. Wiesel (1959) Receptive fields of single neurones in the cat's striate cortex. J. Physiol. (Lond.) 148: 574-591.

Hubel, D. H., and T. N. Wiesel (1961) Integrative action in the cat's lateral geniculate body. J. Physiol. (Lond.) 155: 385-398.

Hubel, D. H., and T. N. Wiesel (1962) Receptive fields, binocular interaction and functional architecture in the cat's visual cortex. J. Physiol. (Lond.) 160: 106-154.

Kato, H., P. O. Bishop, and G. A. Orban (1978) Hypercomplex and simple/complex cell classifications in cat striate cortex. J. Neurophysiol. 41: 1071-1095.
Kuffler, S. W. (1953) Discharge patterns and functional organization of mammalian retina. J. Neurophysiol. 15: 37-68.

Lee, B. B., V. Virsu, and O. D. Creutzfeldt (1977) Responses of cells in the cat lateral geniculate nucleus to moving stimuli at various levels of light and dark adaptation. Exp. Brain Res. 27: 51-59.

Lee, B. B., A. Elepfandt, and V. Virsu (1981) Phase of responses to sinusoidal gratings of simple cells in cat striate cortex. J. Neurophysiol. 45: 818-828.

Levick, W. R., B. G. Cleland, and M. W. Dubin (1972) Lateral geniculate neurons of cat: Retinal inputs and physiology. Invest. Ophthalmol. Vis. Sci. 11: 302-311.

McIlwain, J. T., and O. D. Creutzfeldt (1967) Microelectrode study of synaptic excitation and inhibition in the lateral geniculate nucleus of the cat. J. Neurophysiol. 30: 1-21.

Orban, G. A., H. Kato, and P. O. Bishop (1979) End-zone region in receptive fields of hypercomplex and other striate neurons in the cat. J. Neurophysiol. 42: 818-832.

Rodieck, R. W., and J. Stone (1965) Response of cat retinal ganglion cells to moving visual patterns. J. Neurophysiol. 28: 819-832.

Rose, D. (1979) Mechanisms underlying the receptive field properties of neurons in cat visual cortex. Vision Res. 19: 533-544.

Sillito, A. M., and V. Versiani (1977) The contribution of excitatory and inhibitory inputs to the length preference of hypercomplex cells in layers II and III of the cat's striate cortex. J. Physiol. (Lond.) 273: 775-790.

Singer, W., and N. Bedworth (1973) Inhibitory interaction between $\mathrm{X}$ and $\mathrm{Y}$ units in the cat lateral geniculate nucleus. Brain Res. 49: 291-307.

Singer, W., and O. D. Creutzfeldt (1970) Reciprocal lateral inhibition of on- and off-center neurones in the lateral geniculate body of the cat. Exp. Brain Res. 10: 311-330.

Singer, W., E. Pöppel, and O. D. Creutzfeldt (1972) Inhibitory interaction in the cat's lateral geniculate nucleus. Exp. Brain Res. 14: 210-226.

So, Y. T., and R. Shapley (1981) Spatial tuning of cells in and around lateral geniculate nucleus of the cat: $X$ and $Y$ relay cells and perigeniculate interneurons. J. Neurophysiol. 45: $107-120$

Vidyasagar, T. R., and J. V. Urbas (1982) Orientation sensitivity of cat LGN neurones with and without inputs from visual cortical areas 17 and 18. Exp. Brain Res. 46: 157-169. 